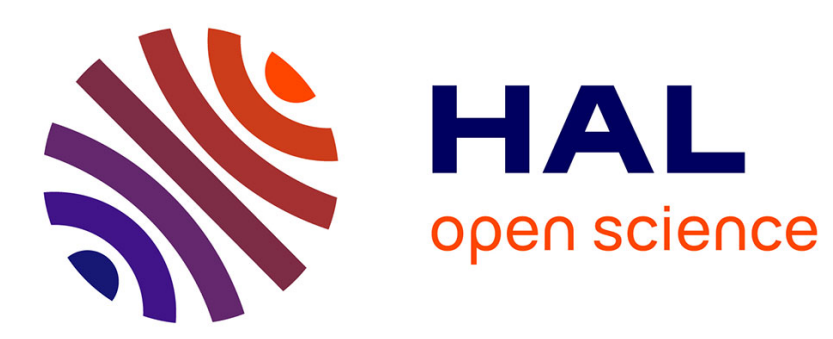

\title{
Assessment of the Urbanization Effect on Seismic Response
}

Claude Boutin, Pierre Roussillon

\section{To cite this version:}

Claude Boutin, Pierre Roussillon. Assessment of the Urbanization Effect on Seismic Response. Bulletin of the Seismological Society of America, 2004, 94 (1), pp.251-268. hal-00941053

\section{HAL Id: hal-00941053 \\ https://hal.science/hal-00941053}

Submitted on 3 Feb 2014

HAL is a multi-disciplinary open access archive for the deposit and dissemination of scientific research documents, whether they are published or not. The documents may come from teaching and research institutions in France or abroad, or from public or private research centers.
L'archive ouverte pluridisciplinaire HAL, est destinée au dépôt et à la diffusion de documents scientifiques de niveau recherche, publiés ou non, émanant des établissements d'enseignement et de recherche français ou étrangers, des laboratoires publics ou privés. 


\title{
Assessment of the Urbanization Effect on Seismic Response
}

\author{
by Claude Boutin and Pierre Roussillon
}

\begin{abstract}
This article introduces two analytical methods that aim at investigating the effect of the urban environment on seismic motions. We regard cities as a periodic distribution of simple oscillators. The response to a vertical incident $S H$ wave is analyzed by considering the city with a macroscopic point of view. As a first approach, the effect of buildings is described by a mean impedance on the soil-city interface. This enables us to identify a mechanical soil-city coupling parameter that governs the magnitude of the interaction and to determine a characteristic time of the soil-city response. A second, more refined model based on approximated wave fields radiated by each building leads to introducing a boundary layer to describe the multiple interactions effect. This provides an assessment of both free soil motion and building basement motion. Applications are given for monofrequency and multifrequency cities in the case of homogeneous and stratified substratums.
\end{abstract}

\section{Introduction}

The seismic risk in urbanized areas deserves particular attention for many reasons, among which are the concentration of population, of real estates, of human activities having a large socioeconomical impact, and the presence of strategic public services.

Numerous studies have addressed the seismic response of cities' settlement sites. The usual approach is to consider that the ground motion results from the seismic source and the structure of the substratum. However, even when using $2 \mathrm{D}$ or $3 \mathrm{D}$ models and refined subsoil descriptions, some features of seismic records remain unexplained. This is the case for the 1985 Michoacan earthquake, for which the long duration of motion in Mexico City (Singh and Ordaz, 1993) and the presence of beatings in some records resist the usual modeling (Chavez-Garcia and Bard, 1994).

Such observations led Wirgin and Bard (1996) to suggest that the response depends not only on the substratum but also on the "surstratum," that is, the city. This idea is supported by the fact that Mexico City is densely urbanized, the natural frequencies of buildings and the basin may coincide for a class of high-rise buildings, and finally, the clay softness is favorable to soil-structure interactions. Then, the energy transmitted to the buildings and reradiated in their surroundings through multiple soil-structure (Jennings and Bielak, 1973; Housner, 1994) and structure-soil-structure interactions (Luco and Contesse, 1973) could modify the ground motion. Simulations performed on idealized cities brought qualitative confirmation of this assumption (Wirgin and Bard, 1996). Recent calculations based on different descriptions of the city (2D, 3D, periodic or nonperiodic) and on various methods (volume eigenmodes, Green functions, finite elements) gave more quantitative results (Clouteau and Aubry, 2001; Guéguen et al., 2002; Semblat et al., 2002;
Tsogka and Wirgin, 2003). They proved that for specific situations, the effect of the city may be important. However, although some trends can be inferred, systematic investigations coupled with a statistical treatment of the results should be carried out in order to identify the (combined) influence of the numerous involved parameters.

Apart from those numerical results, we propose an alternative approach to assess the city's influence. Our aim is to identify the parameters that determine the city effect and to quantify the extent of this phenomenon from measurable criteria. This study does not pretend to provide an exact model but only to give estimates. The analysis proceeds by scale change from the local building scale to the global city scale: from the description of a representative part of the city, a mean behavior is derived, which enables us to characterize the effect at the city scale. Coming back to the local scale, the effects within the city are assessed on average.

In the section on average surface loading approximation, substituting a macroscopically equivalent boundary condition for the city enables us to identify the leading parameters of the soil and the city and to depict the nature of phenomena. In the section on the boundary layer approximation, we take advantage of the cone-wave model commonly used in soil-structure interaction (Meek and Velestos, 1974; Wolf, 1994) to build a surface boundary layer. This allows us to take into account the heterogeneous stress distribution on the surface and to bring corrections to the previous description.

Statement of the Considered Site-City Problem and Main Assumptions

This study is devoted to the seismic response, under vertical incident $S$ waves, of a densely urbanized city resting 
on a homogeneous or stratified plane half-space. We consider cities that show some spatial invariance in plan. In other words, the city is characterized by an elementary representative block (ERB) constituted by several 3D buildings. Conveniently, we assume that the $3 \mathrm{D}$ city is a periodic distribution of the same ERB in the two directions of the ground surface. For certain cities, this assumption may be not so far from reality.

The buildings are taken to be linear elastic structures based on rigid shallow foundations, and the study is restricted to the effect of their first mode of oscillation. Thus, each building is described by an equivalent one-degree-offreedom oscillator.

Even in such schematic cities, the seismic response includes the multiple interactions between structures through the soil, and the exact treatment would be extremely complex. However, the complexity is greatly reduced when the surface motions show a characteristic size of variation significantly larger than the characteristic size $l$ of the ERB. This condition of scale separation is fulfilled at sufficiently low frequencies so that the wavelengths are significantly greater than $l$. In this article, the scale separation is systematically assumed.

In practice the frequency range in which the scale separation is valid, which depends on the city organization and soil conditions, can only be roughly estimated. For instance, assuming a characteristic size $l=50 \mathrm{~m}$ for dense urbanized areas, and considering $f$ such that $\lambda>l$, leads to the validity ranges of $0-1.5 \mathrm{~Hz}$ in Mexico City and $0-4 \mathrm{~Hz}$ in Nice (Table 1).

\section{Average Surface Loading Approximation}

The scale separation hypothesis means that the surface motion varies slowly at the ERB scale, and therefore, as a first approximation, every point of an ERB follows the same motion. This enables us to determine the macro-equivalent boundary condition by expressing, at the soil-city interface, the balance between the stress in the soil on the one hand and the equivalent stress exerted by the city on the other hand. The latter is calculated by averaging over the whole
ERB surface the forces generated by the buildings excited by the same motion at their base. This idea is close to that proposed in structural acoustics to investigate the vibration perturbations induced in a large main structure by a multitude of small substructures attached to it (Soize, 1993; Strasberg and Feit, 1996), the main structure being here replaced by the underlying half-space.

This model would be exact if the entire ERB was lying on a thin, perfectly rigid, foundation layer. Thus the present approach implicitly introduces a full and direct coupling between buildings that is stronger than the real coupling through the soil. Consequently, this model tends to enhance the effects of multiple interactions.

This approach provides a macroscopic vision in the sense that the actual stress boundary conditions are only fulfilled on average. For this reason, the momenta that may exist are not accounted for, since they create stresses of zero average. As usual when using homogenization methods (Bensoussan et al., 1978), the macroscopic description is valid at a certain distance from the interface (SanchezPalencia, 1985). In the vicinity of the interface (i.e., within a depth of the order of the ERB size), the exact description would require a corrective term matching the actual boundary conditions (see the Boundary Layer Approximation section). To overcome this point, in the case of stratified substratum, the top layer will be assumed sufficiently thick so that the field at the bedrock interface is reasonably approximated by the macrodescription.

\section{Monofrequency City Lying on Homogeneous Soil}

In this section, a periodic city made of identical buildings resting on a homogeneous soil is considered (Fig. 1). The ERB (of surface $S$ ) includes a single building (of surface $S_{\mathrm{b}}$ ), and the urban density is given by $\theta=S_{\mathrm{b}} / S$. The first mode of the building, represented by an oscillator made of a spring $K$, a mass $M$, and a viscous damper $C$, is characterized by its free undamped resonant frequency $f_{0}$, with $\omega_{0}=2 \pi f_{0}=\sqrt{K / M}$, and its damping ratio $\xi=$ $C /(2 \sqrt{K M})$. The underlying medium is characterized by its density $\rho$, shear modulus $\mu$, undamped velocity $\beta=\sqrt{\mu / \rho}$, and quality factor $q$ so that the complex shear modulus is

Table 1

Parameters of Site City Interaction

\begin{tabular}{|c|c|c|c|c|c||c|c|c|c|c|}
\hline & \multicolumn{4}{|c||}{ Mexico - Roma Norte } & \multicolumn{5}{c|}{ Nice - Gare Sud } \\
\hline Substratum & $\beta(m / s)$ & $\rho\left(k g / m^{3}\right)$ & $q$ & $h(m)$ & $f_{l}(H z)$ & $\beta(m / s)$ & $\rho\left(k g / m^{3}\right)$ & $q$ & $h(m)$ & $f_{l}(H z)$ \\
Layer 1 & 65 & 1400 & 25 & 40 & 0.4 & 200 & 1600 & 25 & 50 & 1 \\
Layer 2 & 600 & 2000 & 50 & $\infty$ & $/$ & 1000 & 2200 & 100 & $\infty$ & $/$ \\
\hline Impedance Ratio & \multicolumn{3}{|c|}{$R=0.07$} & $R^{-1}=13$ & \multicolumn{3}{|c||}{$R=0.16$} & $R^{-1}=6$ \\
\hline \multirow{2}{*}{ City } & \multicolumn{1}{|c|}{$\theta$} & $\sigma^{0}$ & $\xi$ & $\theta$ & $\sigma^{0}$ & $\xi$ \\
& 0.5 & 0.25 & 0.01 or 0.05 & 0.33 & 0.05 & 0.01 or 0.05 \\
\hline
\end{tabular}

City and substratum data from Guégen ef al. (2002). 


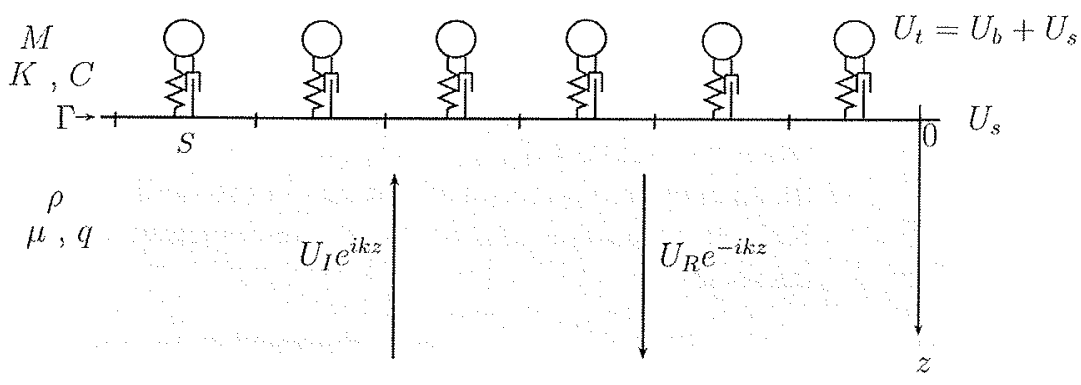

Figure 1. Average surface loading approximation, city resting on homogeneous substratum.

$\mu^{*}=\mu(1+i / q)$. We focus on the response to a harmonic $S$ wave of normal incidence (Fig. 1). As the problem is linear, all the elements oscillate at the incident wave frequency $f=\omega / 2 \pi$. The soil-city interface $\Gamma$ is taken as the origin of the downward vertical axis $O_{z}$. The transverse displacements of the incident and reflected $S$ wave take the form $U_{\mathrm{I}} \exp [i(k z$ $+\omega t)]$ and $U_{\mathrm{R}} \exp [i(-k z+\omega t)]$, respectively, where $i=$ $\sqrt{-1}$ and $k=(1-i / 2 q) \omega / \beta$ is the complex wavenumber. The associated shear stresses (on a surface of normal $-\vec{e}_{z}$ ) are $-\mu^{*} i k U_{\mathrm{I}} \exp [i(k z+\omega t)]$ and $\mu^{*} i k U_{\mathrm{R}} \exp [i(-k z+$ $\omega t)]$. The displacement at the soil-city interface $\Gamma$ is denoted by $U_{\mathrm{s}} \exp [i \omega t]$. As for the building motions, $U_{\mathrm{t}} \exp [i \omega t]$ and $U_{\mathrm{b}} \exp [i \omega t]$ are respectively the total mass displacement and the relative displacement with respect to the basis, so that $U_{\mathrm{t}}=U_{\mathrm{b}}+U_{\mathrm{s}}$.

This problem is governed by three linear equations (the term $\exp [i \omega t]$ is omitted):

- the dynamic balance of the mass,

$$
-K U_{\mathrm{b}}-C i \omega U_{\mathrm{b}}=-M \omega^{2}\left(U_{\mathrm{b}}+U_{\mathrm{s}}\right)
$$

- the displacement continuity on $\Gamma$,

$$
U_{\mathrm{s}}=U_{\mathrm{I}}+U_{\mathrm{R}}
$$

- the balance of the shear stress in the soil and the average shear stress applied on $\Gamma$ (of normal $\vec{n}=-\vec{e}_{z}$ ) by the city,

$$
K U_{\mathrm{b}}+C i \omega U_{\mathrm{b}}=-\mu^{*} i k\left(U_{1}-U_{\mathrm{R}}\right) S
$$

The building transfer function $B(\omega)$ and the force transfer function $F(\omega)$ (also named reactance), respectively relating the total mass displacement to the basis displacement and the force exerted by the building to the basis displacement, take the form

$$
\begin{aligned}
& B(\omega)=\frac{U_{\mathrm{t}}}{U_{\mathrm{s}}}=\frac{\omega_{0}^{2}+2 i \xi \omega \omega_{0}}{\omega_{0}^{2}+2 i \xi \omega \omega_{0}-\omega^{2}}, \\
& F(\omega)=B(\omega) M \omega^{2} .
\end{aligned}
$$

Eliminating $U_{\mathrm{b}}$, the set (equations $2-3$ ) reads

$$
\begin{aligned}
& U_{\mathrm{I}}+U_{\mathrm{R}}=U_{\mathrm{s}}, \\
& U_{\mathrm{I}}-U_{\mathrm{R}}=P(\omega) U_{\mathrm{s}},
\end{aligned}
$$

where the complex function $P(\omega)$ and the parameter $\sigma$, which, as $B(\omega)$ and $F(\omega)$, will be used afterward (with the notations $P, B, F)$, are given by

$$
P(\omega)=\left(1-\frac{i}{2 q}\right) \sigma \frac{i \omega}{\omega_{0}} B(\omega), \quad \sigma=\frac{\sqrt{K M}}{S \sqrt{\mu \rho}} .
$$

The solution leads to the sought waves and motion amplitudes:

$$
\begin{gathered}
U_{\mathrm{s}}=\frac{2 U_{\mathrm{I}}}{1+P}, \quad U_{\mathrm{R}}=U_{\mathrm{I}} \frac{1-P}{1+P} \\
U_{\mathrm{t}}=\frac{2 U_{\mathrm{I}}}{1+P} B, \quad U_{\mathrm{b}}=\frac{2 U_{\mathrm{I}}}{1+P}(B-1) .
\end{gathered}
$$

The total reflection, in the case of the free surface without a city, is recovered when $P(\omega)=0$.

\section{Analysis of the Monofrequency City Effect}

The previous results show that the city effect is described by $P(\omega)$, which plays the role of a complex-valued, frequency-dependent, impedance ratio between the soil and the city.

A Parameter to Estimate the City Influence. The amplitude of $P(\omega)$ linearly depends on the dimensionless parameter $\sigma=\sqrt{K M} /(S \sqrt{\mu \rho})$. Thus the presence of the city is negligible when $\sigma$ tends to zero, which corresponds to soft, light and dispersed structures ( $K \rightarrow 0, M \rightarrow 0, S \rightarrow \infty$ ) built on rigid soils $(\mu \rightarrow \infty)$. Inversely, the city effect is all the more important as the city is densely urbanized, constituted by heavy and rigid buildings, and settled on soft subsoil.

An estimation of $\sigma$ can be derived through usual assumptions. By introducing the building density $\rho_{\mathrm{b}}$ and height $H_{\mathrm{b}}$, their mass is expressed by $M=\rho_{\mathrm{b}} S_{\mathrm{b}} H_{\mathrm{b}}$. For framed structures, the first eigenfrequency is empirically related to the height by $1 / f_{0}=T_{\mathrm{b}} \approx H_{\mathrm{b}} / 30$ (in SI units). Then $\sigma$ is assessed by 


$$
\sigma=\frac{\sqrt{K M}}{S \sqrt{\mu \rho}}=\frac{1}{S} \frac{M}{\rho} \sqrt{\frac{K}{M}} \sqrt{\frac{\rho}{\mu}} \approx 2 \pi \frac{S_{\mathrm{b}}}{S} \frac{\rho_{\mathrm{b}}}{\rho} \frac{30}{\beta}=\sigma^{0} .
$$

The density $\rho_{\mathrm{b}}$ is of the order of $250-350 \mathrm{~kg} / \mathrm{m}^{3}$ and the soil shear velocity $\beta$ ranges between 50 and $1500 \mathrm{~m} / \mathrm{sec}$, so that $\sigma^{0}$ usually lies in between $10^{-2}$ and $10^{-1}$ for very dense cities $(\theta=1 / 2)$. Thus, for real metropolises, $\sigma$ should never exceed 1. For instance, from the Mexico and Nice data (Guéguen et al., 2002) (Table 1), we obtain $\sigma_{\text {Mexico }}^{0} \approx 0.25$ and $\sigma_{\text {Nice }}^{0} \approx 0.05$.

The parameter $\sigma$ differs from the indicator $\varepsilon$ proposed by Guéguen et al. (2002) in the case of a top layer (equation 11). Let us mention that for a homogeneous half-space (top layer of infinite depth), $\varepsilon=0$ whatever the value of $\sigma$ is.

Frequency Dependence of the City Influence. The real part of the building reactance $F(\omega)$ (i.e., the resistive, nondissipative component) is positive below resonance and negative above (Strasberg and Feit, 1996). Thus, below its eigenfrequency the city behaves as an additional mass and above it as an additional spring. Within the scale separation frequency range, the low- and high-frequency approximations (relatively to $f_{0}$ ) of $P(\omega)$ read

$$
\begin{aligned}
& P(\omega) \approx \sigma \frac{i \omega}{\omega_{0}}\left(1-\frac{i}{2 q}\right) \rightarrow 0 \text { when } \omega / \omega_{0} \rightarrow 0, \\
& P(\omega) \approx \sigma 2 \xi\left(1-\frac{i}{2 q}\right) \text { when } \omega / \omega_{0} \rightarrow \infty .
\end{aligned}
$$

The city effect vanishes for frequencies significantly smaller than the building resonance, and, since $\xi \ll 1$ and $q \gg 1$, a slight effect remains at significantly larger frequencies.

In the vicinity of $\omega_{0}$, the city effect is very significant:

$$
P(\omega) \rightarrow \frac{\sigma}{2 \xi}(1+2 i \xi)\left(1-\frac{i}{2 q}\right) \approx \frac{\sigma}{2 \xi} \text { when } \omega / \omega_{0} \rightarrow 1,
$$

and therefore

$$
U_{\mathrm{s}} \approx 2 I \frac{2 \xi}{\sigma+2 \xi}, \quad U_{\mathrm{b}} \approx 2 I \frac{-i}{\sigma+2 \xi} .
$$

At the building resonance, the city acts as a medium of large impedance, $\sigma / 2 \xi$, which decreases the soil-city interface motion as well as the base and internal motions of buildings. The building damping has a strong influence, whereas the effect of soil damping is of second order (this still holds with multiple reflections in a top layer; see the section on a monofrequency city lying on a stratified half-space). For the frequency range of interest and realistic values of $\sigma$ and $\xi$ (Table 1), Figure 2 presents the variations of $U_{\mathrm{s}}$ versus the dimensionless frequency $\omega / \omega_{0}$. On the spectrum response, it can be seen that
- the city decreases the amplitude of $U_{\mathrm{s}}$ whatever the frequency;

- the effect and the frequency range of influence increases when $\sigma$ increases ( $\sigma_{\text {Mexico }}^{0}>\sigma_{\text {Nice }}^{0}$ );

- the maximum of perturbation occurs in the vicinity of the building resonance, and its magnitude increases when $\xi$ decreases.

The effect in time domain is illustrated by the response to a Ricker incident wave of central frequency $f_{\mathrm{R}}$ coinciding with the building frequency $f_{0}$, that is

$$
\hat{U}_{\mathrm{I}}(t)=\left[\left(\pi \frac{t-t_{\mathrm{s}}}{t_{\mathrm{p}}}\right)^{2}-1 / 2\right] \exp \left[\left(\pi \frac{t-t_{\mathrm{s}}}{t_{\mathrm{p}}}\right)^{2}\right],
$$

where $t_{\mathrm{p}}=2 \pi / \omega_{0}$. The presence of the city increases the signal duration. This point is discussed later.

Characteristic Time of the City Influence. Let us determine the time response of the soil-city system to an incident impulsion wave, that is, $\hat{U}_{\mathrm{I}}(t)=\delta(t)$ and $U_{\mathrm{I}}(\omega)=1$, in the case of an undamped soil. We focus on the difference $U_{\mathrm{d}}$ of the soil response in the presence $\left(U_{\mathrm{s}}\right)$ and in the absence $\left(2 U_{\mathrm{I}}\right)$ of a city. According to equation (6) and introducing the function $J(\omega), U_{\mathrm{d}}$ is given by

$$
U_{\mathrm{d}}=U_{\mathrm{s}}-2 U_{\mathrm{I}}=2 U_{\mathrm{I}} \frac{-\sigma}{1+2 \xi \sigma}\left[-2 \xi \omega^{2}+i \omega \omega_{0}\right] J(\omega),
$$

where

$$
\begin{aligned}
J(\omega) & =\frac{1}{-\omega^{2}+2 i \zeta \omega \Omega_{0}+\Omega_{0}^{2}}, \\
\Omega_{0} & =\frac{\omega_{0}}{\sqrt{1+2 \xi \sigma}}, \quad \zeta=\frac{\xi+\sigma / 2}{\sqrt{1+2 \xi \sigma}} .
\end{aligned}
$$

In the time domain, $\hat{U}_{\mathrm{d}}(t)$ is related to $\hat{J}(t)$, the inverse Fourier transform of $J(\omega)$, by

$$
\frac{\hat{U}_{\mathrm{d}}(t)}{\omega_{0}}=2 \frac{-\sigma}{1+2 \xi \sigma}\left[\frac{2 \xi}{\omega_{0}} \ddot{\hat{J}}(t)+\dot{\hat{J}}(t)\right],
$$

where, according to the fact that $\sigma$ and then $\zeta$ are less than $1, \hat{J}(t)$ is given by

$$
\hat{J}(t)=\frac{1}{\Omega_{0} \sqrt{1-\zeta^{2}}} \sin \left[\Omega_{0} \sqrt{1-\zeta^{2}} t\right] \exp \left[-\zeta \Omega_{0} t\right]
$$

Instead of an instantaneous response (in the case of the free surface), the city induces a delayed response even for an undamped substratum. This response shows damped oscillations of pulsation $\Omega_{0}$ close to $\omega_{0}$, a characteristic duration time $1 / \zeta \Omega_{0}$ inversely proportional to $\sigma$ and $\omega_{0}$, and an amplitude proportional to $\sigma$ (when $\xi$ is neglected). These results agree with the calculations presented in Figure 2, where the 

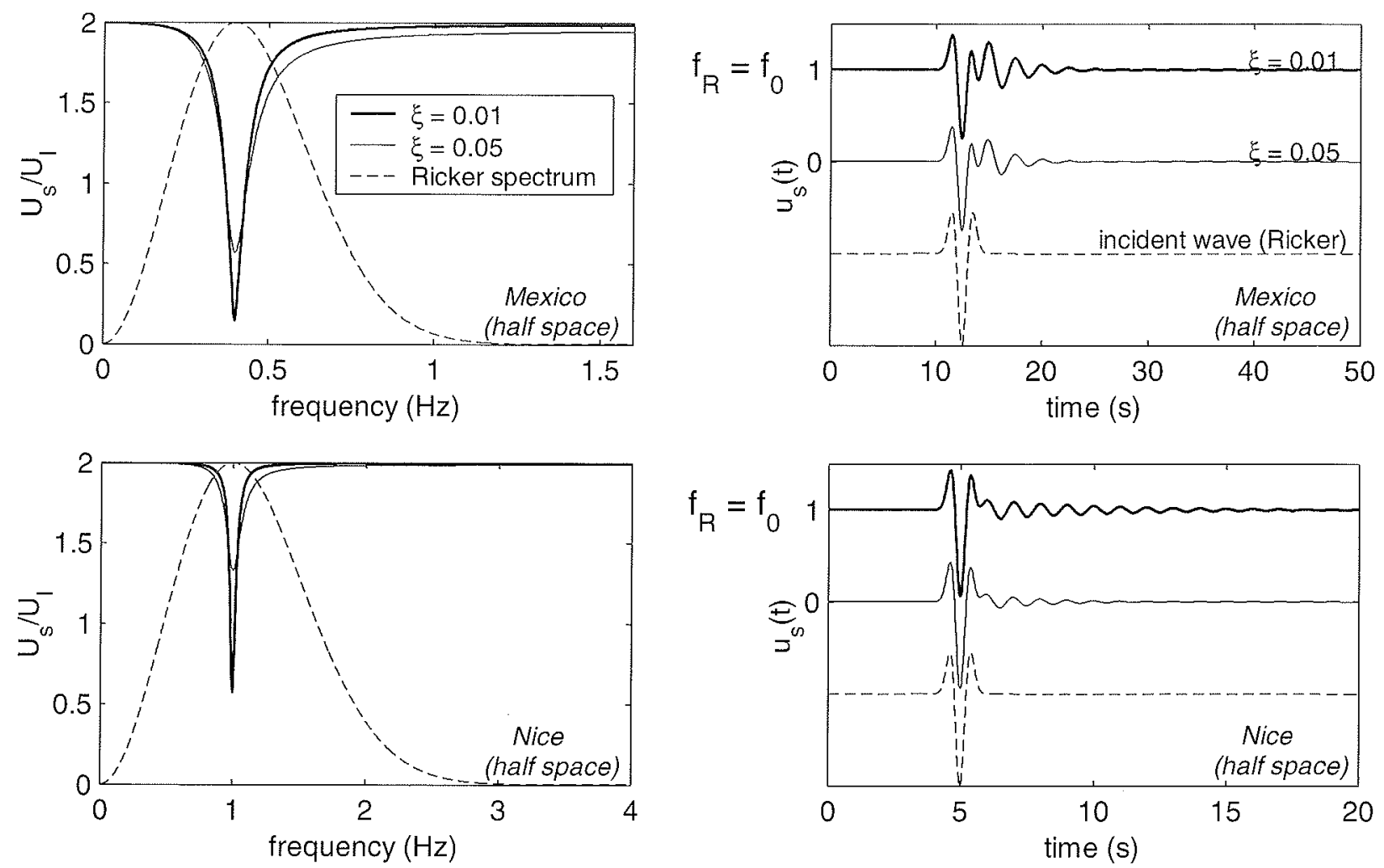

Figure 2. Monofrequency city resting on homogeneous substratum. Left: Spectral response of the overall soil motion $U_{\mathrm{s}}$ to unit vertically incident harmonic $S H$ wave. Dashed line: Ricker spectrum used for time response. Right: Time response to a Ricker wavelet of central frequency identical to building resonant frequency $f_{0}$. Top: Data of the first layer of Mexico City. Bottom: Data of the first layer of Nice.

time duration is larger for Nice than for Mexico City (in half-space configuration) while $\sigma_{\text {Mexico }}^{0}>\sigma_{\text {Nice. Even if they }}^{0}$ cannot be straightly applied in the presence of a top layer, they confirm that the city tends to increase the duration of the soil motion.

From equation (7), the corresponding building motion is derived:

$$
\hat{U}_{\mathrm{b}}(t)=\frac{-2}{1+2 \xi \sigma} \ddot{\hat{J}}(t)
$$

\section{Monofrequency City Lying on Stratified Half-Space}

A city lying on a half-space with one top layer (indexed by 1 ) of thickness $h$ (rigidity $\mu_{1}$, density $p_{1}$, quality factor $\left.q_{1}\right)$ over a bedrock $\left(\mu_{2}, \rho_{2}, q_{2}\right)$ is now considered. $R=$ $\sqrt{\mu_{1} \rho_{1} / \mu_{2} \rho_{2}}$ is the soil-bedrock impedance ratio, and $R^{*}$ includes the damping effects $R^{*}=R\left(1+i / 2 \mathrm{q}_{1}\right) /\left(1+i / 2 \mathrm{q}_{2}\right)$.

Without the city, this configuration induces a site effect (Aki, 1988): for soil of smaller impedance than bedrock $(R<1)$, the surface motion is amplified by a factor $2 / R$ (neglecting damping) at the eigenfrequencies of the layer. Under a normal incident wave, the fundamental frequency corresponds to the quarter wavelength resonance of the layer, that is $\omega_{1}=\pi \beta_{1} / 2 h$, the higher frequencies being given by $\omega_{\mathrm{ln}}=(2 n-1) \omega_{1}$.

In presence of a city, the four equations (displacement and stress continuity on the soil-city and soil-bedrock interfaces) corresponding to an incident vertical $S$ wave take the form $\left(U_{i}^{+}, U_{i}^{-}\right.$, and $k_{i}$, respectively denote the up and down wave amplitude and the wavenumbers in layer $i=1$, 2) (see Fig. 3)

$$
\begin{gathered}
U_{1}^{+}+U_{1}^{-}=U_{\mathrm{sl}}, \\
U_{1}^{+}-U_{1}^{-}=P(\omega) U_{\mathrm{sl}}, \\
U_{1}^{+} \exp \left(i k_{1} h\right)+U_{1}^{-} \exp \left(-i k_{1} h\right)= \\
U_{2}^{+} \exp \left(i k_{2} h\right)+U_{2}^{-} \exp \left(-i k_{2} h\right), \\
R^{*}\left[U_{1}^{+} \exp \left(i k_{1} h\right)-U_{1}^{-} \exp \left(-i k_{1} h\right)\right]= \\
U_{2}^{+} \exp \left(i k_{2} h\right)-U_{2}^{-} \exp \left(-i k_{2} h\right) .
\end{gathered}
$$

It follows that, for an incident wave of amplitude $I=U_{2}^{+}$ $\exp \left(i k_{2} h\right)$, the motion $U_{\mathrm{sl}}$ on $\Gamma$ is

$$
U_{\mathrm{sl}}=2 I \frac{2}{\left(1+R^{*}\right)(1+P) \exp \left(i k_{1} h\right)+\left(1-R^{*}\right)(1-P) \exp \left(-i k_{1} h\right)} .
$$

The city modifies the spectrum response of the free top layer (which would be recovered if $P=0$ ), particularly in the 


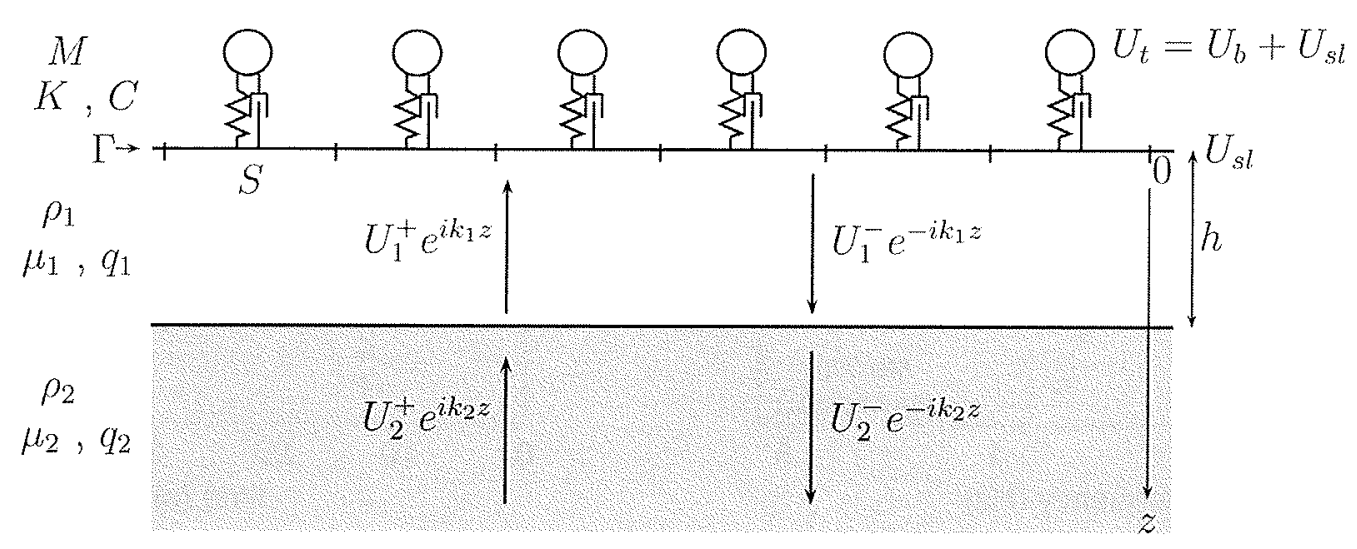

Figure 3. Average surface loading approximation, city resting on stratified substratum.

vicinity of the building resonance where $P(\omega)$ reaches its maximum amplitude. Once more, the influence is enlarged for higher values of $\sigma / 2 \xi$. At any layer resonant frequency $\omega_{\mathrm{ln}}$, the surface motion amplitude is $2 /\left\|R^{*}+P\left(\omega_{\mathrm{ln}}\right)\right\|$. In the undamped case $\left(q_{\mathrm{i}}=\infty ; \xi=0\right)$, it can be proved that the city reduces the maximum amplification of the free top layer and shifts the amplification frequency.

This is illustrated in Figures 4 and 5, which depict $U_{\mathrm{sl}}$ according to the frequency for several ratios of dimensionless pulsation $\omega_{0} / \omega_{\mathrm{I}}$ (or frequency $f_{0} / f_{1}$ ) using Mexico City and Nice data (Table 1). The city influence is larger in Mexico City (Fig. 4) than in Nice (Fig. 5), in accordance with the smaller value of $\sigma$ in Nice.

The main spectrum perturbations occur when the building and layer resonance frequencies coincide (for instance, $\omega_{0} / \omega_{1}=1$ or 3 ): instead of a simple peak, a double peak of lower amplitude appears. At the coinciding frequency, neglecting the weak damping, the amplitude of the transfer function is estimated by $2 /(R+\sigma / 2 \xi)$ instead of $2 / R$ in the absence of a city. If the building resonance occurs at a noamplification frequency of the layer, the city induces an additional asymmetric peak $\left(\omega_{0} / \omega_{1}=1 / 2,3 / 2\right)$ and/or the shift of the dominant frequencies (for instance, $\omega_{0} / \omega_{1}=2$ ). According to the mass or springlike effect of the building, one sytematically observes that the layer resonant frequencies higher (respectively lower) than the eigenfrequency of the building are shifted to lower (respectively higher) frequencies. The increase of building damping smooths the perturbations of the transfer functions.

The time responses to a Ricker incident wave of central frequency $f_{\mathrm{R}}=f_{0}$ (equation 9) show a systematic increase of the signal duration, particulary significant for asymmetric peaks. The double peaks in the spectra produce beatings in the signals. However, for wide spectrum incident motion, the influence of buildings far from the layer resonance will be masked by the dominant effect of the layer amplification. Thus the perturbation will be mainly due to buildings of frequency close to that of the layer.

Guéguen et al. (2002) proposed a city influence indicator $\varepsilon$ based on the kinematic energy in the soil and city, assuming that the layer essentially oscillates at its resonant frequency and the building frequency is larger than that of the layer. For a monofrequency city, $\varepsilon$ takes the form

$$
\varepsilon=\theta \frac{\rho_{\mathrm{b}}}{\rho} \frac{H_{\mathrm{b}}}{h}\left[\frac{\omega_{1}}{\omega_{0}}\right]^{2}
$$

while, with the same parameters, $\sigma$ can be expressed as

$$
\sigma=\frac{1}{S} \frac{M}{\rho} \sqrt{\frac{K}{M}} \sqrt{\frac{\rho}{\mu}}=\theta \frac{\rho_{\mathrm{b}}}{\rho} \frac{H_{\mathrm{b}}}{h} \frac{\omega_{0}}{\omega_{\mathrm{I}}} \pi / 4
$$

The comparison of $\varepsilon$ and $\sigma$ shows that both have similar values when $\omega_{0} / \omega_{1} \approx 1$. The different physical meaning of both parameters may explain why they diverge at other frequencies.

\section{Generalization to Multifrequency Cities}

For multifrequency periodic cities, the ERB is made of $N$ different buildings (indexed by $n$ ) distributed on the surface $S$. The urban density related to buildings $n$ is given by $\theta_{n}=S_{\mathrm{b} n} / S$, and the urban density is $\theta=\sum_{1}^{N} \theta_{n}$. All the previous building parameters are now indexed by $n$.

When studying the response to a vertically incident harmonic $S$ wave, the only change between a mono- and a multifrequency city appears in the stress balance equation (3), where $P$ is replaced by $P_{M}$, which involves the contributions of all the ERB's buildings:

$$
P_{\mathrm{M}}(\omega)=\left(1-\frac{i}{2 q}\right) \sum_{n=1}^{N} \sigma_{n} \frac{i \omega}{\omega_{0 n}} B_{n}(\omega), \sigma_{n}=\frac{\sqrt{K_{n} M_{n}}}{S \sqrt{\mu \rho}}
$$

Using the same argument as for monofrequency cities, we have

$$
\sigma_{\mathrm{n}} \approx \frac{\theta_{n}}{\theta} \sigma^{\mathrm{o}}
$$



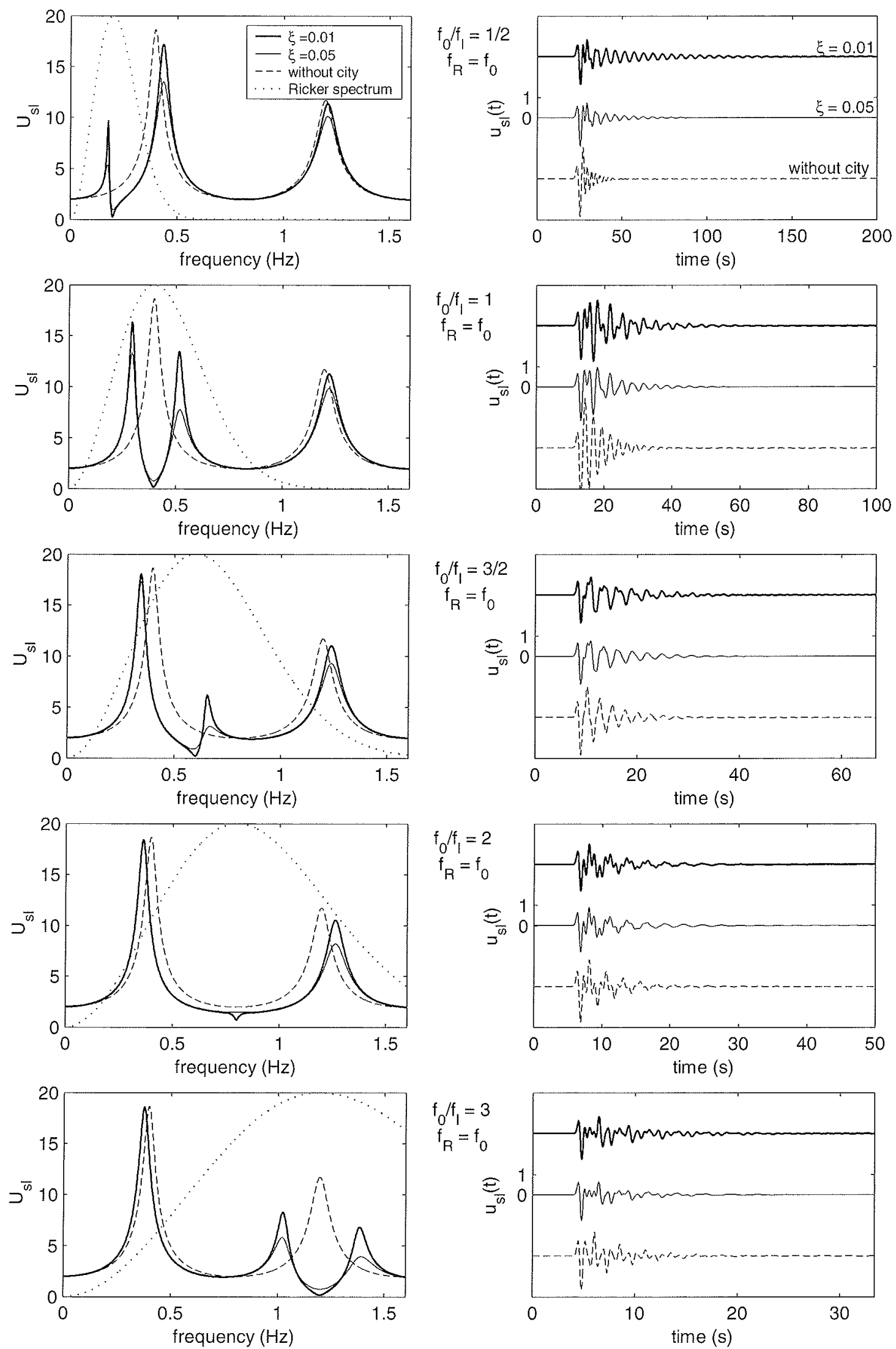

Figure 4. Monofrequency cities resting on stratified substratum. Mexico City data. From top to bottom: ratio of resonance frequency of building to that of the layer equal to $1 / 2,1,3 / 2,2,3$. For other comments, see Figure 2 . 

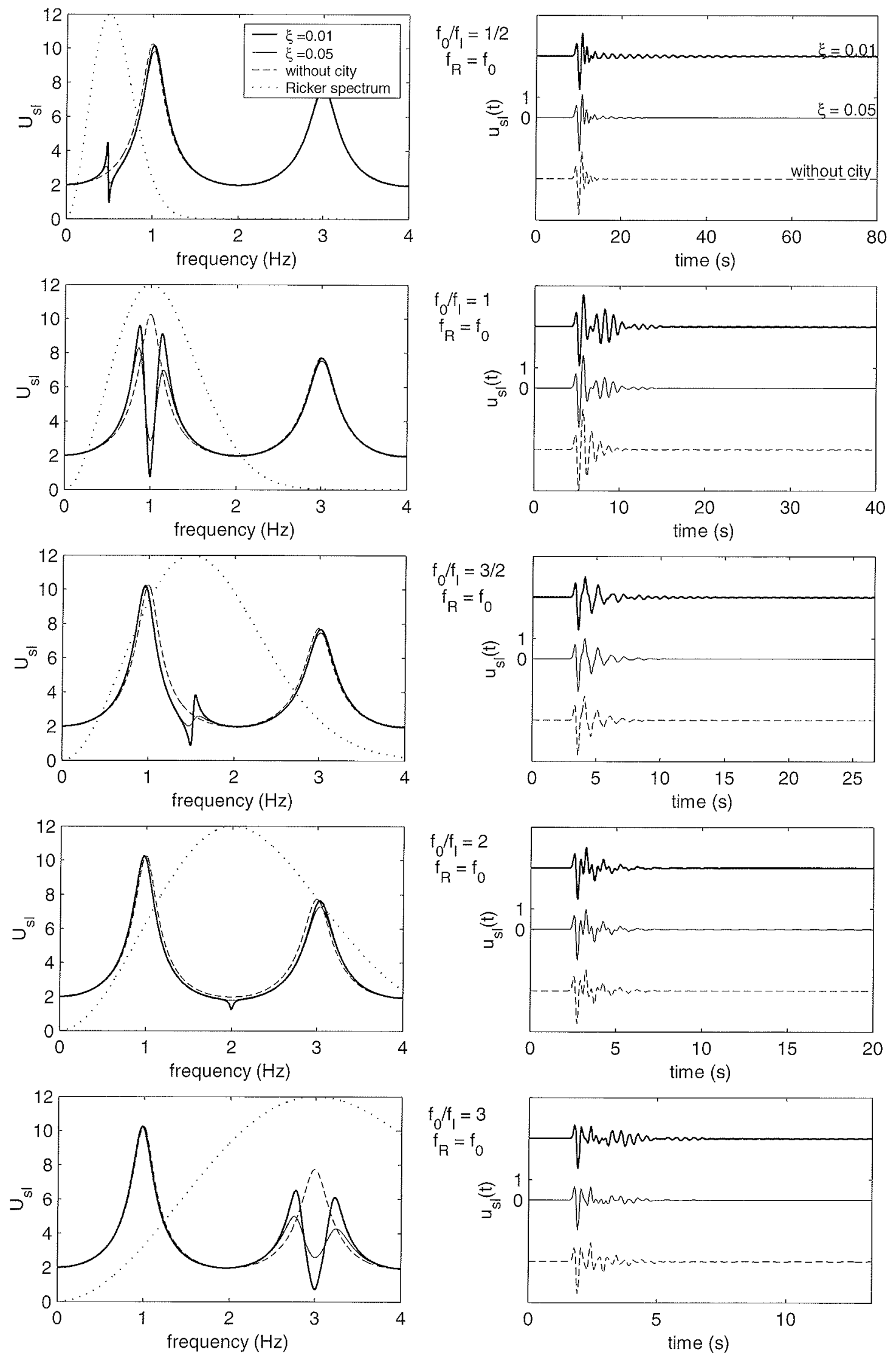

Figure 5. Same as Figure 4, with Nice data. 
The soil motion and the amplitude of the reflected wave are now given by

$$
U_{\mathrm{sM}}=\frac{2 U_{\mathrm{I}}}{1+P_{\mathrm{M}}}, \quad U_{\mathrm{RM}}=U_{\mathrm{I}} \frac{1-P_{\mathrm{M}}}{1+P_{\mathrm{M}}}
$$

whereas the internal and total motions are different for each building:

$$
U_{\mathrm{t} n}=\frac{2 I}{1+P_{\mathrm{M}}} B_{n}, \quad U_{\mathrm{b} n}=\frac{2 I}{1+P_{\mathrm{M}}}\left(B_{n}-1\right) .
$$

As the $P_{\mathrm{M}}$ properties are linked to those of $P_{n}$ functions, the spectrum perturbation is spread over the whole range of building frequencies, and since $\sigma_{n}<\sigma^{\circ}$, its amplitude is less than for a monofrequency city of the same $\sigma^{\circ}$. This appears when comparing the spectrums in Figure 2 and Figure 6. The latter was obtained for cities made of five kinds of buildings $\left(\theta_{n} / \theta=1 / 5\right)$ with eigenpulsations: $\omega_{0 n} / \omega_{1}=1 / 2,1$, $3 / 2,2,3$. Thus, the city influence is more important for cities made up of nearly identical buildings than for buildings of dispersed frequency.

From the high- and low-frequency approximations of $P_{\mathrm{M}}$, two characteristic times of the impulsive response $\tau_{0}$ and $\tau_{\infty}$, related to the exponential decay respectively at short and long duration, can be derived. Neglecting the weak soil and structure damping, they read

$$
\tau_{0}=\frac{1+2 \sum_{n=1}^{N} \sigma_{n} \xi_{n}}{\sum_{n=1}^{N} \sigma_{n} \omega_{0 n}}, \quad \tau_{\infty}=\frac{\sum_{n=1}^{N} \frac{1+2 \sigma_{n} \xi_{n}}{\omega_{0 n}^{2}}}{\sum_{n=1}^{N} \frac{\sigma_{n}}{\omega_{0 n}}} .
$$

The city effect in the presence of a top layer is derived by substituting the function $P_{\mathrm{M}}$ for $P$ in equation (10) to obtain $U_{\text {sIM }}$ and using, for the total and internal motions of buildings $n$,

$$
U_{\mathrm{t} n}=U_{\mathrm{sIM}} B_{n}, \quad U_{\mathrm{b} n}=U_{\mathrm{slM}}\left(B_{n}-1\right)
$$

The perturbation is rather complex; however the main effect, once more, is due to buildings having a resonant frequency close to that of the layer (see Fig. 7 beside Figs. 4 and 5 for $f_{0}=f_{1}$ ). The response to a Ricker incident wave of central frequency coinciding with the layer frequency shows that the beats in the signals may be even more marked than for monofrequency cities. Indeed, the weaker effect can make the twin peaks closer.

As a conclusion, we see that the average surface loading approximation enables us to identify the features of the city effect through a mean impedance of the city. Nevertheless,
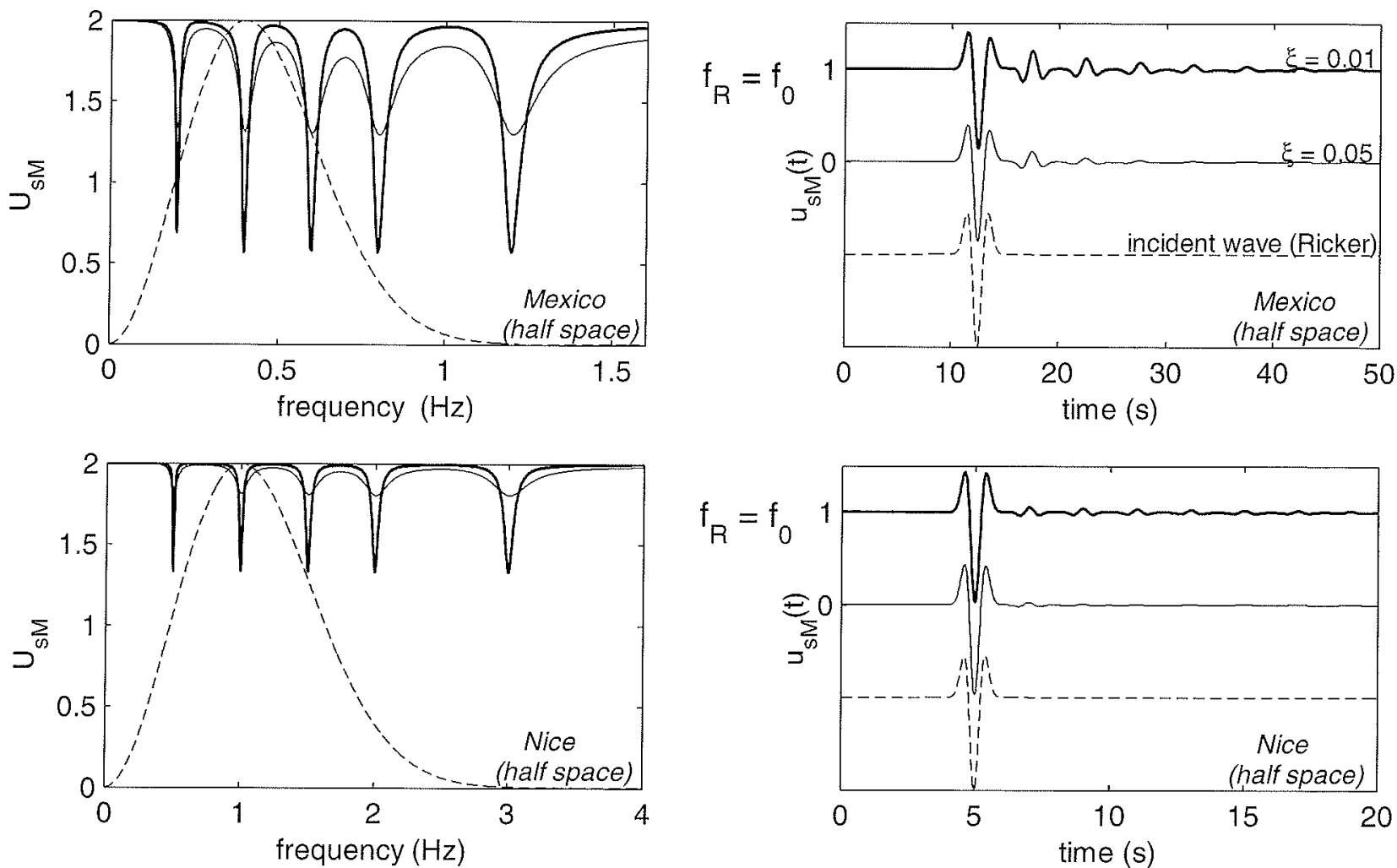

Figure 6. Multifrequency city resting on homogeneous substratum. City composed of equidistributed buildings of resonant frequency $f_{0} / 2, f_{0}, 3 f_{0} / 2,2 f_{0}, 3 f_{0}$. For other comments, see Figure 2. 
this approach is limited by the fact that the boundary conditions on the surface are only fulfilled on average. Moreover the global motion obtained for the whole city area does not account for the disparity of motions between the building foundations and the actual "free" surface.

\section{Boundary Layer Approximation}

To improve the previous study, it is necessary to take into account the toothlike stress distribution on the soil-city interface (constant under buildings, zero outside). The exact determination of the field radiated by such conditions could be handled numerically; however the identification of the role of the parameters requires a fair number of simulations.

An alternative approach, allowing an explicit expression of the results, is presented. The idea is to define a boundary layer achieving the transition from the heterogeneous stresses at the soil-city interface to homogeneous stresses at a certain depth. For this purpose, we use the soil-structure interaction (SSI) cone model, which approximates the field radiated by a shallow foundation and gives analytical expressions of dynamic foundation impedances (Meek and Velestos, 1974).

To lighten the presentation, soils are assumed elastic; the addition of damping would be obtained by introducing a quality factor in the rigidity.
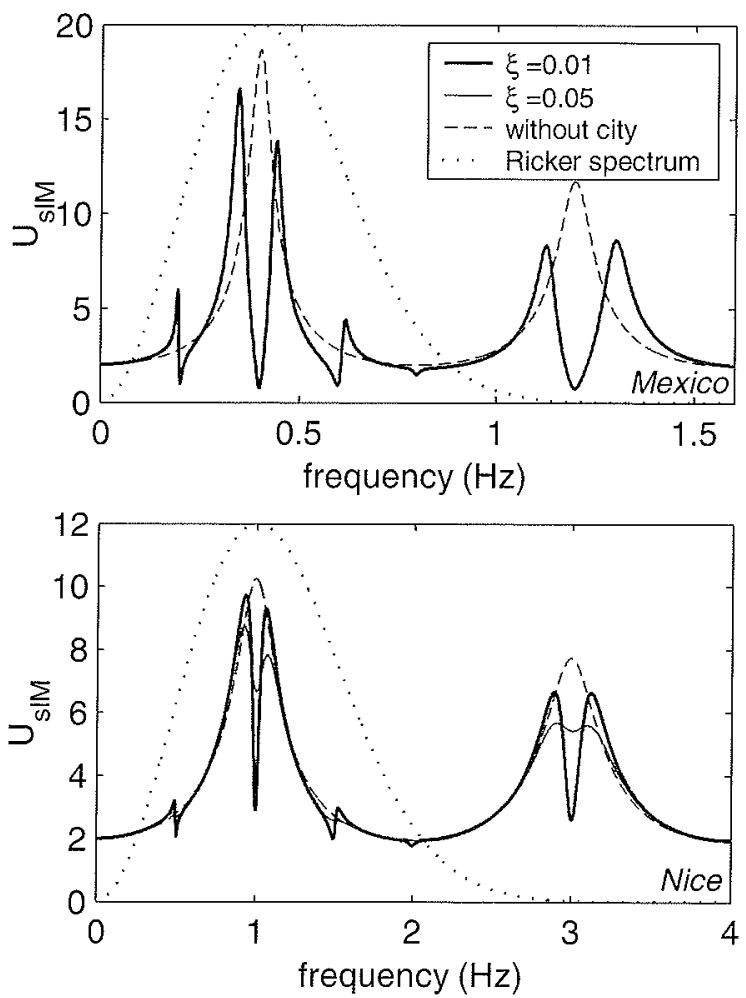

\section{Basics of the Cone Model}

Let us introduce the cone model in the case of a rigid circular shallow foundation of radius $r_{0}$ resting on a halfspace of shear rigidity $\mu$ and loaded by an harmonic horizontal force $T_{0} \exp [i \omega t]$ (Wolf, 1994). The approach is based on three main assumptions:

- The wave field is confined in a truncated cone of vertical axis passing through the foundation center (Fig. 8). The cone apex, at height $z_{0}$ above the ground, is determined by equating in static $(\omega=0)$ the exact impedance to the cone model impedance.

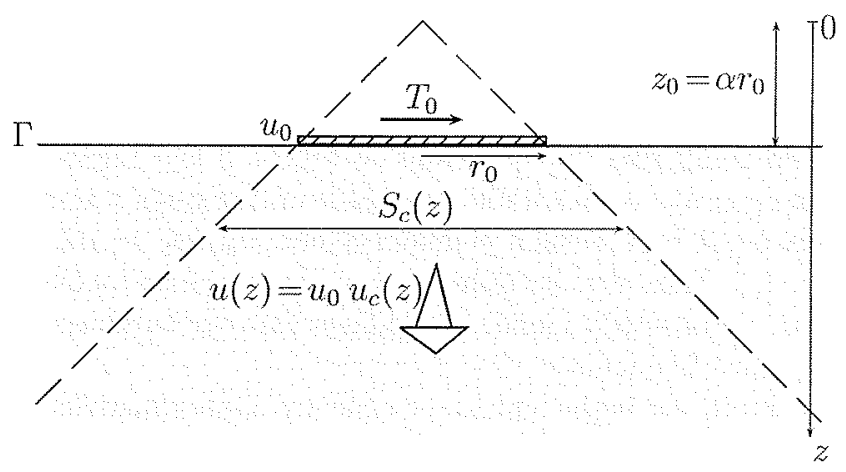

Figure 8. Cone model wave field.
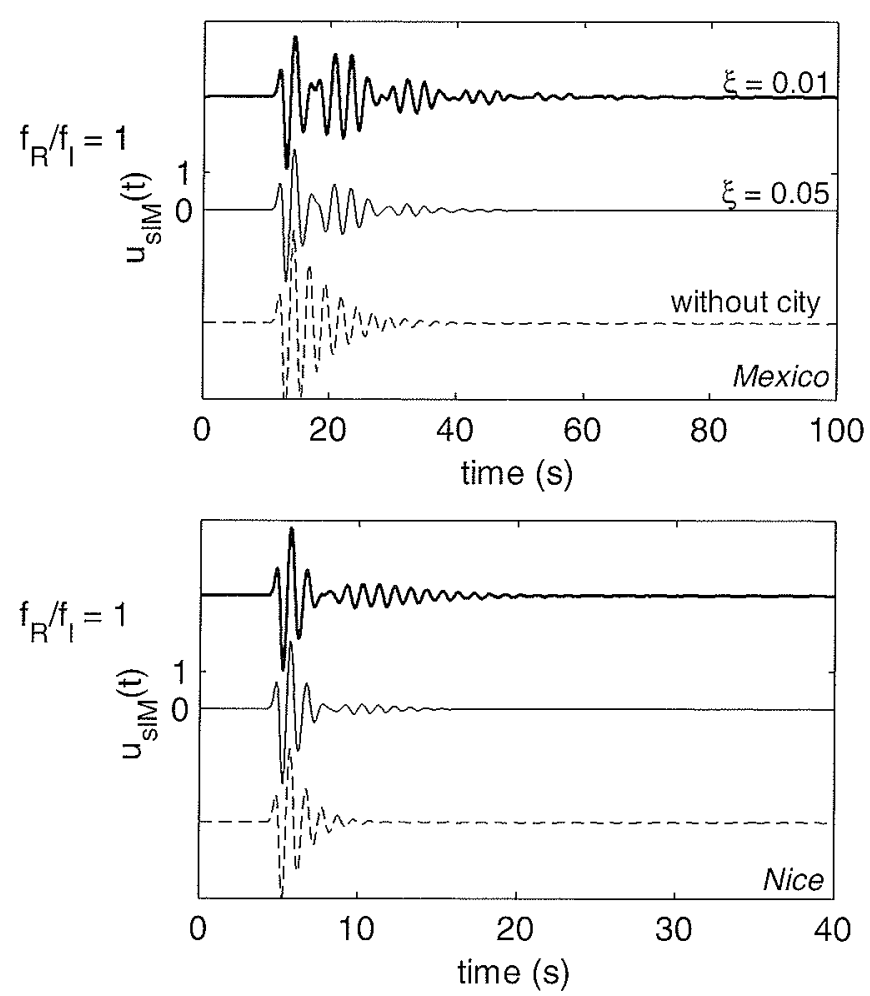

Figure 7. Multifrequency city resting on stratified substratum. Same city as Figure 6. Top: Mexico City data. Bottom: Nice data. Other comments as in Figure 2. 
- Only the stress balance and the components in the direction of the loading are considered, that is, the horizontal displacement $u$ and the tangential stress $\tau$.

- These variables depend only on the depth $z$. According to the elastic behavior of the soil, both are related by

$$
\tau(z)=\mu \frac{d}{d z} u(z)
$$

In the following, the apex is taken as the origin of the vertical axis. The cone section at the depth $z$ is $S_{\mathrm{c}}(z)=$ $\left(z / z_{0}\right)^{2} S_{\mathrm{c}}\left(z_{0}\right)$, and $S_{\mathrm{c}}\left(z_{0}\right)=S_{0}$ is the surface of the foundation. In the loading direction, the balance of forces applied to a thin cone section reads

$$
\frac{d}{d z}\left[\tau(z) S_{\mathrm{c}}(z)\right]=-\rho \omega^{2} u(z) S_{\mathrm{c}}(z)
$$

and leads for $z u$ to the $1 \mathrm{D}$ shear-wave propagation equation

$$
\mu \frac{d^{2}}{d z}[z u]=-\rho \omega^{2} z u .
$$

Considering the downward cone wave $(k=\omega / \beta)$, the motions and stresses in the cone are

$$
\begin{aligned}
u(z) & =u_{0} u_{\mathrm{c}}(z), \quad u\left(z_{0}\right)=u_{0}, \\
u_{\mathrm{c}}(z) & =\frac{z_{0}}{z} \exp \left[-i k\left(z-z_{0}\right)\right], \\
\tau(z) & =-\mu(i k+1 / z) u(z) .
\end{aligned}
$$

Then, on the surface, the force $T_{0}=-S_{0} \tau\left(z_{0}\right)$ is related to the foundation displacement $u_{0}$ by the dynamic impedance $G$ :

$$
G=T_{0} / u_{0}=i \mu k S_{0}\left[1+1 / i k z_{0}\right] .
$$

In static $(k=0)$, the matching of $G$ with the exact impedance imposes

$$
z_{0} / r_{0}=\alpha=\frac{\pi}{8}(2-\nu)
$$

where $v$ is the soil Poisson ratio. The cone angle is quite open: for $v=1 / 3$ the cone aspect ratio is $\alpha=z_{0} / r_{0} \approx 2 / 3$. At sufficiently low frequency, namely $\omega r_{0} / \beta \leq 8$, which is the case in the seismic range for usual structures, comparisons between the numerical solution and cone model approximation (Wolf, 1994) validate the approach. The results obtained for circular foundations can also apply to square foundations of the same area. Thus, this approach supplies an approximated Green function, at least for the motions under the foundation. The weak point of the cone model is the lack of field description outside the cone. For a periodic distribution of foundations, this defect is partially overcome by means of the boundary layer introduced subsequently.

\section{City Lying on Homogeneous Substratum}

We consider an isolated building at first, then a boundary layer is introduced for periodic foundations, and finally the city influence is derived. The substratum is assumed homogeneous.

Isolated Building. From the SSI cone model, the response of an isolated building to a vertical harmonic $S$ wave can be derived. Let us superpose to the wave field in the absence of a building (i.e., upward incident and downward reflected waves of same amplitude $I$ ) the field radiated by the building in the form of a cone-shaped wave of unknown surface amplitude $u_{1}$. By doing so, the free field conditions are respected beside the building foundation. The building foundation motion is given by $U_{\mathrm{SSI}}=2 I+u_{1}$. The force on the building base is only balanced by the cone-shaped field in the soil: $T=G u_{1}$ (equation 15), while, according to the building behavior (equation 4$), T$ is also related to the base motion by $T=F(\omega)\left(2 I+u_{1}\right)$. Equating these two expressions gives $u_{1}$, and the foundation motion is

$$
U_{\mathrm{SSI}}=\frac{2 I}{1-F / G} .
$$

This approximation leaves the free surface motion unmodified by the building: $U_{\Gamma}=2 I$. Finally, the total mass motion and internal building motion are given by

$$
U_{\mathrm{tSSI}}=B(\omega) U_{\mathrm{SSI}}, \quad U_{\mathrm{bSSI}}=(B(\omega)-1) U_{\mathrm{SSI}} .
$$

Boundary Layer for Periodic Distribution of Foundations. Attention is now focused on the approximated field resulting from a $2 \mathrm{D}$ periodic distribution of identical foundations loaded by the same synchronized forces, $T_{0} \exp [i \omega t]$. For this purpose, we define a boundary layer adapted to a periodic toothlike surface stress distribution. Conveniently, the equivalent radius $r_{0}$ of the foundation $\left(S_{0}=\pi r_{0}^{2}=S_{\mathrm{b}}\right)$ will be used. The cone-shaped fields radiated by the foundations cover the whole substratum surface at a depth $Z$ determined by the cone aspect ratio $\alpha$ and the urban density $\theta$ (Fig. 9):

$$
\left(\frac{z_{0}}{Z+z_{0}}\right)^{2}=\theta, \quad Z=(1 / \sqrt{\theta}-1) \alpha r_{0}
$$

$Z$ defines the boundary layer thickness and, at this level, the motion and stress can be looked upon as uniform (neglecting un- and overlapped zones) and given from equations (13) and (14) by

$$
u\left(z_{0}+Z\right)=\frac{z_{0}}{z_{0}+Z} u_{0} \exp [-i k Z]
$$




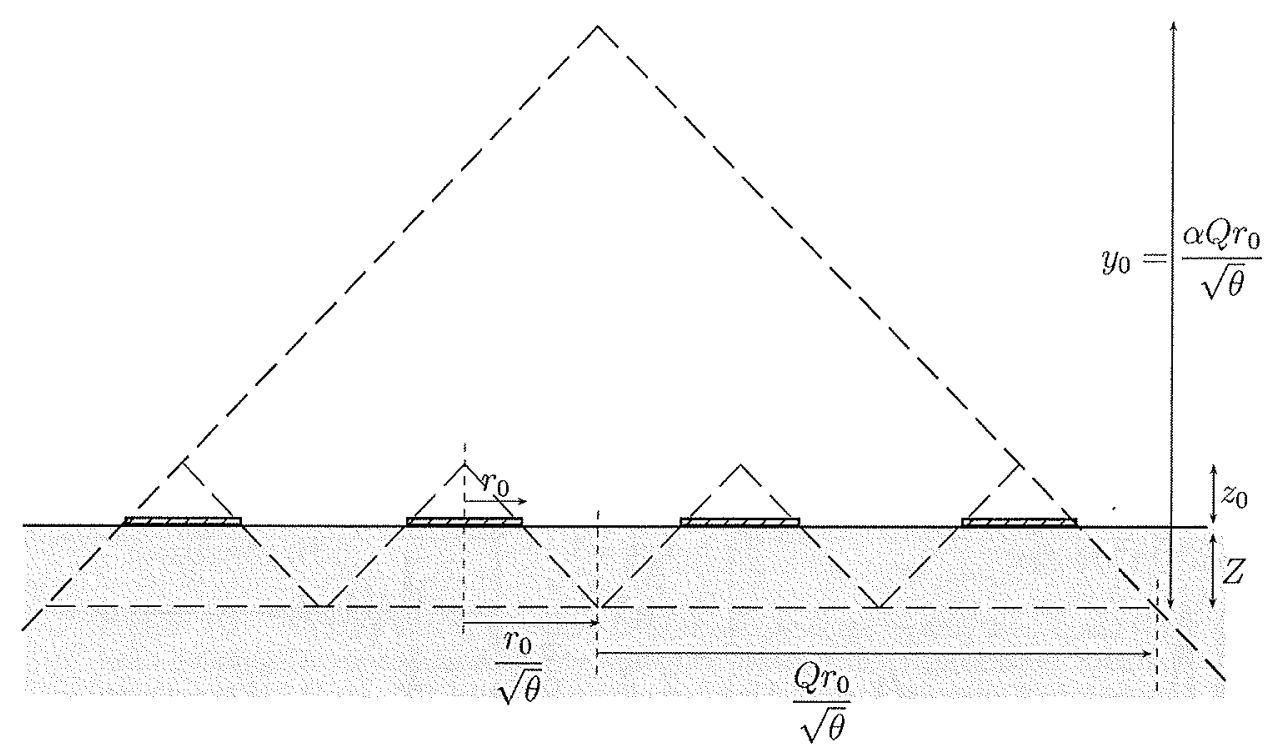

Figure 12. Boundary layer for a city of finite extension.

$z_{0}$, and $Z$ are related by

$$
\begin{aligned}
y=z+y_{0}-\left(Z+z_{0}\right) & \\
\frac{z_{0}}{y_{0}} & =\frac{\sqrt{\theta}}{Q}, \frac{y_{0}-Z}{y_{0}}=\frac{Q-1+\sqrt{\theta}}{Q} .
\end{aligned}
$$

The motions and stresses due to the diffracted cone fields (scaled by $u_{0}$ ) read, respectively,

$$
\begin{gathered}
C^{+}(y)=C_{0}^{+} \frac{y_{0}}{y} \exp \left[i k\left(y-y_{0}\right)\right], C^{-}(y)=C_{0}^{-} \frac{y_{0}}{y} \exp \left[-i k\left(y-y_{0}\right)\right], \\
\tau^{+}(y)=\mu(i k-1 / y) C^{+}(y), \quad \tau^{-}(y)=-\mu(i k+1 / y) C^{-}(y) .
\end{gathered}
$$

The amplitudes $C_{0}^{+}, C_{0}^{-}$are determined by expressing that

- on the free surface $\Gamma\left(y=y_{0}-Z\right)$, $\tau^{+}\left(y_{0}-Z\right)+\tau^{-}\left(y_{0}-Z\right)=0$

- at the depth $Z$ from the ground surface $\left(y=y_{0}\right)$, the total field is balanced by a single downward cone wave propagated below $Z$, written $C_{\mathrm{E}}(y)=C_{\mathrm{E} O} \frac{y_{0}}{y} \exp \left[-i k\left(y-y_{0}\right)\right]$.

These three requirements lead to the following set of equations:

$$
\begin{aligned}
-\left(i k+\frac{1}{y_{0}-Z}\right) \exp [i k Z] C_{0}^{-}+\left(i k-\frac{1}{y_{0}-Z}\right) \exp [-i k Z] C_{0}^{+}=0, \\
-\left(i k+\frac{1}{y_{0}}\right) C_{0}^{-}+\left(i k-\frac{1}{y_{0}}\right) C_{0}^{+}-\left(i k+\frac{1}{Z+z_{0}}\right) \frac{z_{0}}{Z+z_{0}} \exp [-i k Z]=-\left(i k+\frac{1}{y_{0}}\right) C_{\mathrm{E} 0}, \\
C_{0}^{-}+C_{0}^{+}+\frac{z_{0}}{Z+z_{0}} \exp [-i k Z]=C_{\mathrm{E} 0},
\end{aligned}
$$
tions.

The comparison of $U_{0 \mathrm{FE}}$ with $u_{0} \tilde{U}_{0}=(1+2 D) u_{0}$ (equation 22) shows that both are close when $\sqrt{\theta} /(Q-1+\sqrt{\theta}) \ll$ $k z_{0}$ and $Q \gg 1$. In practice, the results would be very similar

for $Q>5$ and when the size of the city $Q \alpha r_{0} / \sqrt{\theta}$ is larger than one or two wavelengths. For instance, the Mexico Roma Norte and Nice-Gare Sud zones are about $1 \mathrm{~km}^{2}$ and $50 \mathrm{~m}$ and can be taken for the ERB size so that $Q \approx 20$. The infinite city assumption would give a reasonable approximation for frequencies larger than $2 \beta / 1000 \approx 0.15 \mathrm{~Hz}$ (Mexico City) and $2 \beta / 1000 \approx 0.4 \mathrm{~Hz}$ (Nice).

This boundary field could be used to determine the finite city response. Incidentally it provides the foundation impedance of structures based on a network of shallow founda-

and the resolution gives the foundation motion: 


\section{Conclusion}

The macroscopic approach gives an assessment of the urbanization effect on the seismic motions, from accessible data on the soil and the city. The main phenomena, that is, the increase of duration, amplitude perturbation, and beating of signals, are derived analytically and the leading parameters identified. It is shown how the site and city effects may combine and modify the free-field site response. The average loading approximation gives the mean uniform response within the city. The boundary layer provides separate estimates of the foundation motions of each kind of building and a mean value for the remaining free ground. The influence of the geometrical building distribution on the ERB and the local fluctuations of the free soil motion are not described.

These results enable to us forecast the situations in which the city (or wide industrial areas with heavy facilities) may contaminate the records. The interest lies in the analysis of seismic or ambient noise recordings in urban areas or in the consequences for vulnerability analysis. The city effect can arise even in the case of a homogeneous substratum, and it will be stronger when coinciding with a site effect. However, clear experimental evidence would be difficult to obtain. Indeed, the nature (anomalies spread over the whole building frequency range) and the magnitude of the contamination are such that, in most configurations, the ground motion will apparently be primarily controlled by the site effect. However, in favorable situations (Mexico City) the frequency content and the time decrease of recordings coda should give indications.

Finally, let us come back to the main limitations of the approach and discuss them briefly. The first restriction lies in the frequency range of validity. To investigate the reliability of the main assumptions used in this article, let us consider again the response of a periodic city (of square period $l \times l)$ to a vertically incident $S H$ wave. The city periodicity enforces the same periodicity of stresses and motions on the soil-city interface $\Gamma$, which act as a 2D periodic distribution of sources. Thus the $S H$ field radiated by each periodic point source can be decomposed in a discrete series of plane $S H$ waves on the functional basis:

$$
\begin{aligned}
& \exp [(2 i \pi m / l) x] \exp [(2 i \pi n / l) y] \exp \left[i k_{m, n} z\right] m, n \text { integers } \\
& \text { with }(m 2 \pi / l)^{2}+(n 2 \pi / l)^{2}+k_{m, n}^{2}=(\omega / \beta)^{2}=(2 \pi f / \beta)^{2}
\end{aligned}
$$

The whole sources induces a diffracted $S H$ wave field on the form

$$
\sum_{m=-\infty}^{m=\infty} \sum_{n=-\infty}^{n=\infty} A_{m, n} \exp [(2 i \pi m / l) x] \exp [(2 i \pi n / l) y] \exp \left[i k_{m, n} z\right]
$$

The term $m=n=0$ corresponds to a homogeneous plane $S H$ wave propagating vertically $\left(k_{0,0}=\omega / \beta\right)$. Its amplitude $A_{0,0}$ is related to the first term of the Fourier series, that is, the average value, of the source distribution on $\Gamma$. The nature of the higher terms depends on $/$ compared to the wavelength $\lambda=\beta l f$.

- If $l<\lambda$, that is, for frequency $\omega<2 \pi \beta / l$ belonging to the scale separation range, then $\left(k_{m, n}\right)^{2}<0$ for $m n \neq 0$. These fields vanish as the depth increases, and the far diffracted field consists of the vertical $S H$ wave resulting from the mean loading on $\Gamma$. This is consistent with the assumptions used in this article, although it does not give a complete justification.

- If the scale separation is not fulfilled, $l>\lambda$, a finite number of higher terms are propagative and these homogeneous plane waves must be integrated in the far diffracted field. The diffracted wave field is much more complex and the macroscopic approach breaks down.

The second restriction is the periodicity assumption. Very likely, within the scale separation frequency range, this hypothesis is convenient to identify the main phenomena even for a real, disordered city (presenting some spatial invariance). This idea is supported by the fact that long wavelengths cannot discern small objects, which are only perceived as a whole. Inversely, at short wavelengths the diffraction phenomena are very different in ordered and disordered media and the periodic city assumption is questionable. Then, in real cities the effect within the scale separation frequency range can be assessed without exact knowledge of the buildings' distribution. At higher frequencies the coherence of the diffracted field is weakened (or lost) and the local building distribution becomes predominant. Nevertheless, if the city effect is weak at low frequency, strong coupling at high frequencies (except local structure-soilstructure interactions) is improbable.

The third restriction concerns the simplifications introduced in the wave fields and building motions for which only the antiplane motions are considered, which may only partially describe the phenomena. Nevertheless, the agreement (at least qualitative) of the conclusions of the study with aforementioned numerical simulations leads us to think that those simplifications are acceptable to obtain credible estimates.

\section{Acknowledgments}

This research was supported by the French CNRS program ACICATNAT.

\section{References}

Aki, K., (1988). Local site effects on strong ground motion, II: Recent advances in ground motion evaluation, J. L. Von Thun (Editor), Geotechnical Special Publication No. 20, American Society of Civil Engineers, New York, 103-155.

Bensoussan, A., J. L. Lions, and G. Papanicolaou (1978). Asymptotic Analysis of Periodic Structures, North-Holland, Amsterdam.

Chavez-Garcia, F. J., and P. Y. Bard (1994). Site effects in Mexico City eight years after the September 1985 Michoacan earthquake, Soil Dyn. Earthquake Eng. 13, 229-247. 
Clouteau, D., and D. Aubry (2001). Modification of ground motion in dense urban areas, J. Comput. Acoust. 6, 1659-1675.

Guéguen, P., P.-Y. Bard, and F. J. Chavez-Garcia (2002). Site-city seismic interaction in Mexico City-like environements: an analytic study, Bull. Seism. Soc. Am. 92, 794-804.

Housner, G. W. (1994). Interaction of building and ground during an earthquake, Soil Dyn. Earthquake Eng. 13, 229-247.

Jennings, P. C., and J. Bielak (1973). Dynamics of building-soil interaction, Bull. Seism. Soc. Am. 63, 9-48.

Luco, J. E., and L. Contesse (1973). Dynamic structure-soil-structure interaction, Bull. Seism. Soc. Am. 62, 63-83.

Meek, J., and A. Velestos (1974). Simple model for foundations in lateral and rocking motions, in Proc. Fifth World Conference on Earthquake Engineering, Rome, Vol. 2, IAEE, 2610-2613.

Sanchez-Palencia, E. (1985). Homogenisation Techniques for Composite Media, Springer, New York.

Semblat, J. F., P. Guéguen, M. Kham, and P.-Y. Bard (2002). Site-city interaction at local and global scale, in Proc. Twelfih European Conference on Earthquake Engineering, Elsevier, Amsterdam (CD. ROM).

Singh, S. K., and M. Ordaz (1993). On the origin of the long coda observed in lake-bed strong-motion records of Mexico City, Bull. Seism. Soc. Am. 83, 1298-1306.
Soize, C. (1993). A model and numerical method in the medium frequency range for vibroacoustic predictions using theory of structural fuzzy, J. Acoust. Soc. Am. 94(2), 849-865.

Strasberg, M., and D. Feit. (1996). Vibration damping of large structures induced by attached small resonant structures, J. Acoust. Soc. Am. 99(1), 335-344.

Tsogka, C., and A. Wirgin (2003). Simulation of seismic response in an idealized city, Soil Dyn. Earth. Eng. 23, 391-402.

Wirgin, A., and P.-Y. Bard (1996). Effects of buildings on the duration and amplitude of ground motion in Mexico City, Bull. Seism. Soc. Am. 86(3), 914-920.

Wolf, J. P. (1994). Foundation Vibration Analysis using Simple Physical Models, Prentice Hall, New York.

Laboratoire Geomatériaux

DGCB URA CNRS 1652

Ecole Nationale des Travaux Publics de l'Etat

Rue Maurice Audin

69518 Vaulx-en-Velin, France

claude.boutin@entpe.fr, roussillon@entpe.fr 